Supporting information for

\title{
High-speed Complementary Integrated Circuit with a Stacked Structure using Fine Electrodes Formed by Reverse Offset Printing
}

Yasunori Takeda ${ }^{\dagger *}$, Tomohito Sekine ${ }^{\dagger}$, Yi-Fei Wang ${ }^{\dagger}$, Tomoko Okamoto ${ }^{\ddagger}$, Hiroyuki

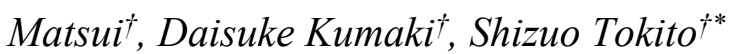

$\dagger$ Research Center for Organic Electronics, Yamagata University, 4-3-16, Jonan, Yonezawa, Yamagata, 992-8510, Japan

\$DIC Corporation, 631, Sakado, Sakura, Chiba, 285-8668, Japan

*Corresponding author: Y. Takeda (y.takeda@yz.yamagata-u.ac.jp) and S. Tokito (tokito@yz.yamagata-u.ac.jp) 
Table S1. Gain characteristics and noise margin depending on the supply voltage of the fabricated inverter circuit.

$\begin{array}{cccc}\text { Supply voltage }(\mathrm{V}) & \text { Gain } & \text { Noise margin }(\mathrm{V}) & \text { Noise margin (\%) } \\ 2.5 & 3 & 0.3 & 24 \\ 5 & 5.6 & 0.84 & 34 \\ 7.5 & 7.5 & 1.41 & 38 \\ 10 & 10 & 1.97 & 39\end{array}$

The noise margin, defined as the size of the largest square that can be inscribed between the input-output characteristics and mirrored characteristics. 


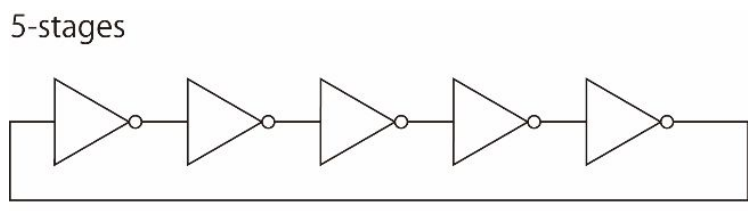

9-stages

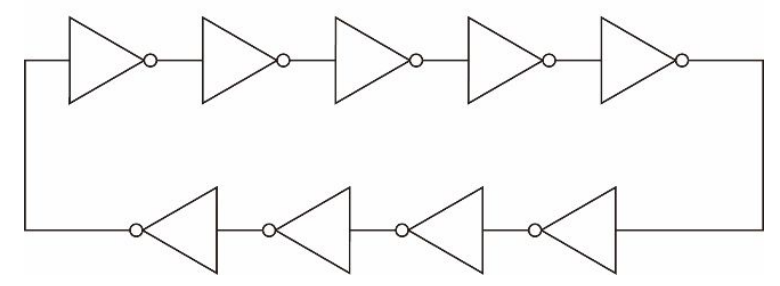

Figure S1. A symbol diagram of the fabricated 5- and 9-stage ring oscillators. 
Table S2. Oscillation frequencies related to the propagation delay time for each stage of the typical 5- and 9-stage ring oscillators.

$$
\text { Deley Time (s) Frequency }(\mathrm{Hz})
$$

Supply Voltage (V) 9-stage RO 5-stage RO 9-stage RO 5-stage RO

$\begin{array}{ccccc}1.25 & 5.79 \mathrm{E}-04 & & 96 & \\ 1.5 & 4.79 \mathrm{E}-04 & & 116 & \\ 2 & 3.55 \mathrm{E}-04 & & 157 & \\ 2.5 & 2.83 \mathrm{E}-04 & 2.28 \mathrm{E}-04 & 197 & 439 \\ 3 & 2.36 \mathrm{E}-04 & 2.12 \mathrm{E}-04 & 235 & 471 \\ 4 & 1.81 \mathrm{E}-04 & 1.75 \mathrm{E}-04 & 308 & 572 \\ 5 & 1.45 \mathrm{E}-04 & 1.43 \mathrm{E}-04 & 384 & 699 \\ 7.5 & 1.04 \mathrm{E}-04 & 9.44 \mathrm{E}-05 & 532 & 1059 \\ 10 & 7.55 \mathrm{E}-05 & 6.82 \mathrm{E}-05 & 736 & 1465 \\ 12.5 & 5.78 \mathrm{E}-05 & 5.17 \mathrm{E}-05 & 962 & 1934\end{array}$




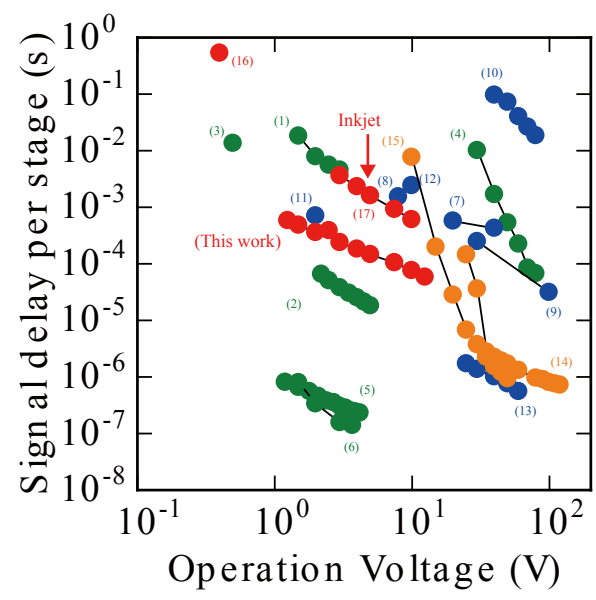

Figure S2. The propagation delay time per stage as a function of the organic ring oscillator operating voltage (green dots: vacuum deposition electrode/semiconductor; blue dots: all-printed; orange dots: printed semiconductor and gate insulator; red dots: printed electrodes and semiconductor).

(1) Sekitani, T.; Zschieschang, U.; Klauk, H.; Someya, T., Flexible organic transistors and circuits with extreme bending stability. Nature Mater. 2010, 9, 1015.

(2) Zschieschang, U.; Ante, F.; Yamamoto, T.; Takimiya, K.; Kuwabara, H.; Ikeda, M.; Sekitani, T.; Someya, T.; Kern, K.; Klauk, H., Flexible Low-Voltage Organic Transistors and Circuits Based on a High-Mobility Organic Semiconductor with Good Air Stability. Adv. Mater. 2010, 22, 982.

(3) Jinno, H.; Yokota, T.; Matsuhisa, N.; Kaltenbrunner, M.; Tachibana, Y.; Someya, T., Low operating voltage organic transistors and circuits with anodic titanium oxide and phosphonic acid self-assembled monolayer dielectrics. Org. Electron. 2017, 40, 58. (4) Zhang, L.; Wang, H.; Zhao, Y.; Guo, Y.; Hu, W.; Yu, G.; Liu, Y., Substrate-Free Ultra-Flexible Organic Field-Effect Transistors and Five-Stage Ring Oscillators. $A d v$. Mater. 2013, 25, 5455.

(5) Ante, F.; Kälblein, D.; Zaki, T.; Zschieschang, U.; Takimiya, K.; Ikeda, M.; Sekitani, T.; Someya, T.; Burghartz, J. N.; Kern, K.; Klauk, H., Contact resistance and megahertz operation of aggressively scaled organic transistors. Small 2012, 8, 73-79.

(6) Borchert, J.W.; Peng, B.; Letzkus, F.; Joachim, N. B.; Chan, P. K. L.; Zojer, K.; Ludwigs, S.; Klauk, H., Small contact resistance and high-frequency operation of flexible low-voltage inverted coplanar organic transistors. Nature Commun. 2019, 10, 1119. 
(7) Fukuda, K.; Takeda, Y.; Mizukami, M.; Kumaki, D.; Tokito, S., Fully SolutionProcessed Flexible Organic Thin Film Transistor Arrays with High Mobility and Exceptional Uniformity. Sci. Rep. 2014, 4, 3947.

(8) Tee, B. C.-K.; Chortos, A.; Berndt, A.; Nguyen1, A. K.; Tom, A.; McGuire, A.; Lin, Z. C.; Tien, K.; Bae, W.-G.; Wang, H.; Mei, P.; Chou, H.-H.; Cui, B.; Deisseroth, K.; $\mathrm{Ng}, \mathrm{T}$. N.; Bao, Z., A skin-inspired organic digital mechanoreceptor. Science 2015, 350, 313.

(9) Mandal, S.; Dell'Erba, G.; Luzio, A.; Bucella, S. G.; Perinot, A.; Calloni, A.; Berti, G.; Bussetti, G.; Duò, L.; Facchetti, A.; Noh, Y.-Y.; Caironi, M., Fully-printed, allpolymer, bendable and highly transparent complementary logic circuits. Org. Electron. 2015, 20, 132.

(10) Huebler, A.C.; Doetz, F.; Kempa, H.; Katz, H.E.; Bartzsch, M.; Brandt, N.; Hennig, I.; Fuegmann, U.; Vaidyanathan, S.; Granstrom, J.; Liu, S.; Sydorenko, A.; Zillger, T.; Schmidt, G.; Preissler, K.; Reichmanis, E.; Eckerle, P.; Richter, F.; Fischer, T.; Hahn, U., Ring oscillator fabricated completely by means of mass-printing technologies. Org. Electron. 2007, 8, 480.

(11) Xia, Y.; Zhang, W.; Ha, M.; Cho, J. H.; Renn, M. J.; Kim, C. H.; Frisbie, D., Printed Sub-2 V Gel-Electrolyte-Gated Polymer Transistors and Circuits. Adv. Funct. Mater. 2010, 20, 578.

(12) Jung, M.; Kim, J.; Noh, J.; Lim, N.; Lim, C.; Lee, G.; Kim, J.; Kang, H.; Jung, K.; Leonard, A. D.; Tour, J. M.; Cho, G., All-Printed and Roll-to-Roll-Printable 13.56MHz-Operated 1-bit RF Tag on Plastic Foils. IEEE Trans. Electron. Dev. 2010, 57, 571.

(13) Mishima, Y.; Akiyama, M.; Hashimoto, K.; Watanabe, N.; Kamata, T., 14-3: High-Performance Organic-TFT Circuits Fabricated by All-Printing Technology on Flexible Plastic Substrates. SID Int. Symp. Dig. Tech. Pap. 2017, 48, 176.

(14) Smith, J.; Hamilton, R.; Heeney, M.; Leeuw, D. M.; Cantatore, E.; Anthony, J. E.; McCulloch, I.; Bradley, D. D. C.; Anthopoulos, T. D., High-performance organic integrated circuits based on solution processable polymer-small molecule blends. Appl. Phys. Lett. 2008, 93, 253301. (15) Kronemeijer, A. J.; Gili, E.; Shahid, M.; Rivnay, J.; Salleo, A.; Heeney, M.; Sirringhaus, H., A Selenophene-Based Low-Bandgap Donor-Acceptor Polymer Leading to Fast Ambipolar Logic. Adv. Mater. 2012, 24, 1558.

(16) Shiwaku, R.; Matsui, H.; Hayasaka, K.; Takeda, Y.; Fukuda, T.; Kumaki, D.; Tokito, S., Printed Organic Inverter Circuits with Ultralow Operating Voltages. $A d v$. Electron. Mater. 2017, 3, 1600557. 
(17) Takeda, Y.; Hayasaka, K.; Shiwaku, R.; Yokosawa, K.; Shiba, T.; Mamada, M.; Kumaki, D.; Fukuda, K.; Tokito, S., Fabrication of Ultra-Thin Printed Organic TFT CMOS Logic Circuits Optimized for Low-Voltage Wearable Sensor Applications. Sci. Rep. 2016, 6, 25714. 
Table S3. XPS surface elemental analysis of the electrode surface after sintering.

Table S3. Atomic Concentration Table (atomic \%)

\begin{tabular}{|lllll|} 
& C & O & F & Ag \\
\hline Only sintering & 50.5 & 15.1 & 10.6 & 23.9 \\
\hline After IPA rinse & 48.8 & 14.4 & 7.2 & 29.5 \\
\hline After N2 plasma & 31.1 & 14.6 & 7.4 & 46.5 \\
\hline
\end{tabular}


Table S4. Propagation delay time per stage of four devices.

\begin{tabular}{|c|c|c|c|c|c|c|}
\hline Vdd $(\mathbf{V})$ & A $(\boldsymbol{\mu s e c})$ & B $(\boldsymbol{\mu s e c})$ & C $(\boldsymbol{\mu s e c})$ & D $(\boldsymbol{\mu s e c})$ & Ave. $(\boldsymbol{\mu s e c})$ & Std. \\
\hline $\mathbf{7 . 5}$ & 97.5 & 94.4 & 104.2 & 104.4 & 100 & 4.29 \\
\hline $\mathbf{1 0 . 0}$ & 70.5 & 68.3 & 74.6 & 75.5 & 72.2 & 2.95 \\
\hline $\mathbf{1 2 . 5}$ & 54.0 & 51.7 & 56.7 & 57.8 & 55.0 & 2.36 \\
\hline $\mathbf{1 5 . 0}$ & 42.9 & 41.4 & 44.9 & 45.5 & 43.6 & 1.65 \\
\hline
\end{tabular}

Std.: standard deviation 
